\title{
Hodgkin-Like Post-Transplant Lymphoproliferative Disorder
}

National Cancer Institute

\section{Source}

National Cancer Institute. Hodgkin-Like Post-Transplant Lymphoproliferative Disorder.

NCl Thesaurus. Code C7239.

A lymphoproliferative disorder with morphologic features resembling Hodgkin lymphoma

that develops in a patient following transplantation. 